\title{
Satisfacción del paciente con tres especialidades dentales
}

\author{
RICARDO MUZA ${ }^{(1)}$ y PAULA MUZA ${ }^{(2)}$
}

\section{RESUMEN}

Las encuestas de la satisfacción del paciente han llegado a ser una herramienta cada vez más importante en la medición de la calidad de los servicios dentales.

El objetivo de este estudio es evaluar el nivel de satisfacción de pacientes mujeres con tres especialidades dentales: odontopediatría, periodoncia y cirugía oral.

Se utilizó una muestra de 238 pacientes mujeres, de 15 años y más que recibieron atención dental al menos dos veces en el servicio dental de Hospital Rancagua de Chile. Fueron entrevistadas durante julio del 2007, acerca de sus experiencias en visitas previas. Las pacientes reportaron el nivel de satisfacción en una escala de 5 puntos tipo Likert para las 10 preguntas en un cuestionario, focalizado en áreas de: acceso, comunicación con el dentista, infraestructura, y otros aspectos.

Las áreas evaluadas menos que regular incluyen la obtención de citaciones en un tiempo determinado, y tiempo de espera en la clínica para acceder al dentista. Las participantes estuvieron regularmente satisfechas con explicaciones del dentista acerca del tratamiento dental, y acceso al tratamiento dental. Lo peor evaluado fue la disponibilidad de baño. El área que recibió la mejor satisfacción por las pacientes fue la competencia técnica del dentista.

Los resultados indican que el nivel de satisfacción del paciente fue moderadamente excelente. Las áreas identificadas como necesitadas de mejoramiento incluyen: comunicación dentistapaciente, sistema de citación, acortar el tiempo de espera para acceder al dentista.

Palabras clave: servicio dental, satisfacción del paciente, calidad de la atención dental.

\section{ABSTRACT}

\section{PATIENT SATISFACTION WITH THREE DENTAL SPECIALTIES}

Patient satisfaction surveys have become an increasingly useful tool for the measurement of the quality of dental services.

The purpose of the present study is to assess the degree of satisfaction of female patients with three dental specialties: odontopediatrics, periodontics and oral surgery.

A 238 female patient sample was used. Patients were aged 15 and more, and had received dental care at least twice at the Dental Service of the Hospital Rancagua, in Chile. They were surveyed during July 2007 and asked about their experience in prior visits. Patients reported the degree of satisfaction in a Likert-like 5 points score, for each of the 10 questions, focused in the fields of : access, communication with the dentist, infrastructure and other aspects.

The areas that resulted as less than average after assessment, included the obtainment of

(1) Jefe Servicio Dental. Hospital Regional Rancagua. Brasil 859 of. 309. Rancagua. Chile.rmuzac@yahoo.com

(2) Psicóloga Clínica, Gendarmería. C. R. S. Santiago. Chile 
appointments in a prescheduled time, and the waiting time at the clinic to gain access the dentist. The participants were in average satisfied with the explanations the dentist gave to them concerning the dental treatment and the access to the dental treatment. The availability of restrooms was the worst assessed. The area receiving the best satisfaction from the patients was the technical skills of the dentist.

Such results indicate that the degree of patient satisfaction was moderately excellent. The areas identified as in need of an improvement include the following: dentist-patient rapport, appointment assignment system, shortening of the waiting time to access the dentist.

Keywords: Dental service, patient satisfaction, dental care quality.

\section{INTRODUCCIÓN}

La calidad de la atención son todas las cualidades con las que cuenta un producto y/o servicio para ser de utilidad a quien se sirva de él. Es decir, un servicio es de calidad cuando sus características, tangibles e intangibles, satisfacen las necesidades y expectativas de sus usuarios.

La prestación de servicios de salud comprende todas las actividades cuyo objetivo fundamental es mejorar, fomentar o restaurar la salud de la población ${ }^{1}$. Si bien estos son los objetivos que siempre han sido el fundamento de los sistemas de salud, en años recientes la Organización Mundial de la Salud (OMS) ha propuesto dos objetivos fundamentales más: el trato adecuado a los usuarios y la justicia financiera ${ }^{1}$.

En la medición de la satisfacción del paciente debemos considerar, entre otros aspectos, que el usuario participe en la toma de decisiones relacionadas con su salud; que el usuario tenga acceso a la información sobre su salud; que el usuario reciba toda la información que requiere sobre su estado de salud y tratamiento; que el usuario reciba un trato humano, goce plenamente de todos sus derechos y no reciba agravios a su dignidad; que el tiempo transcurrido entre la búsqueda de atención y la provisión del servicio no genere riesgos o molestias además de las que ocasionó la búsqueda de atención; que las características físicas de la unidad de servicio, como limpieza, disponibilidad de baños, amplitud e iluminación, sean apropiadas. Estos dominios o factores fueron identificados, y definidos por grupos de expertos por la OMS con el fin explícito de integrar los aspectos más relevantes de la relación de los usuarios con el sistema de salud $^{2}$.

La satisfacción del paciente, en las atenciones de salud en general, ha demostrado la importancia de la confianza del paciente con el profesional, en relación a la calidad de la atención ${ }^{3}$. Esto es muy relevante en todos los aspectos de la atención dental, pero es particularmente importante en situaciones donde la cooperación del paciente es vital, como por ejemplo en la terapia periodontal y en la atención de niños. Que los dentistas conversen y sean comunicativos con los niños es un importante criterio de evaluación por los pacientes ${ }^{4}$. Estudios han reportado desde hace muchos años, la importancia de la comunicación y la entrega de información a los pacientes $^{5,6}$.

Hay estudios que reportan que la satisfacción con la atención dental está fuertemente influenciada con experiencias previas, la importancia de la comunicación y la entrega de información a los pacientes ${ }^{7,8}$. Está demostrado que los pacientes ansiosos están más propensos a estar insatisfechos con la atención dental ${ }^{9,10}$.

Lo mencionado anteriormente nos motivó a realizar este trabajo de satisfacción del paciente con relación a tres especialidades odontológicas, en el servicio dental del Hospital Rancagua.

\section{OBJETIVO}

Evaluar el nivel de satisfacción de pacientes mujeres con tres especialidades dentales: odontopediatría, periodoncia y cirugía oral. 


\section{MATERIAL Y MÉTODO}

Es un estudio descriptivo exploratorio que se realizó mediante una encuesta de opinión, no probabilística, aplicada a 238 pacientes mujeres que acudieron a tratamiento dental durante dos semanas seguidas (lunes a viernes), en julio de 2007, al servicio dental del Hospital de Rancagua, en las especialidades de odontopediatría (78 pacientes), periodoncia (73 pacientes) y cirugía oral (87 pacientes). Se incluyó en esta encuesta a todas las pacientes (niñas y adultas) que habían recibido al menos dos atenciones previas, y que aceptaron voluntariamente contestar la encuesta. Se excluyeron a las pacientes que venían por urgencia, con interconsulta de referencia nueva, a las que ya habían participado en la encuesta en días anteriores para evitar repeticiones, y a las que no dieron su consentimiento (21). La encuesta fue validada previamente con un piloto de 25 pacientes mujeres, en el mismo lugar, y en las mismas condiciones. La opinión fue recogida a través de la aplicación de un cuestionario proporcionado a las pacientes mayores de 15 años, o a sus madres o acompañantes de las menores de 15 años, en la sala de espera previa a su atención dental. Las pacientes reportaron el nivel de satisfacción a 10 preguntas en un cuestionario en forma anónima, focalizado en áreas de acceso, comunicación con el dentista, infraestructura y otros aspectos. Las preguntas tenían cinco posibilidades de respuesta, que fueron excelente, regular, malo, muy malo, no sé. Los aspectos explorados, fueron los siguientes: competencia técnica del dentista; información acerca de su tratamiento dental por el dentista; trato del dentista hacia usted; tiempo de espera en la clínica para acceder al dentista; costo del tratamiento; comunicación con el dentista; acceso al tratamiento dental; limpieza de la sala de espera; obtener citaciones en tiempo determinado y disponibilidad de baños.

Se recogieron las encuestas en un sobre después de haber sido contestadas, por una alumna de la carrera de auxiliar dental de un centro formador externo, que realizaba su práctica en el servicio dental, quien fue capacitada previamente, aplicó el piloto previo de validación, y respondió dudas cuando fue necesario sin inducir la respuesta.

Los datos fueron recogidos en el programa SPSS 12 y realizado su análisis estadístico utilizando Chi-cuadrado de Pearson.

\section{RESULTADOS}

Todas las personas de la muestra respondieron el cuestionario completo.

En la Tabla 1 se muestra la descripción de la muestra por variable edad.

Respecto a la satisfacción con "disponibilidad de baños", la respuesta "muy mal" fue marcada por los pacientes de odontopediatría en $76.9 \%$; de periodoncia en $71.2 \%$; y de cirugía oral en $70.1 \%$. Los resultados muestran que la disponibilidad de baños produce un mayor impacto negativo en los pacientes de odontopediatría. $\mathrm{p}=0.007$; es decir hay significancia estadística.

La satisfacción con "obtener citaciones en un determinado tiempo", la respuesta de pacientes de odontopediatría fue, excelente en $33.3 \%$ y regular en $32.1 \%$. Los pacientes de periodoncia respondieron excelente en $43.8 \%$ y regular en $32.9 \%$. Los pacientes de cirugía oral respondie-

Tabla 1. Distribución de la muestra por edad media, especialidad y total

\begin{tabular}{|l|c|c|c|}
\hline Especialidad & Media & N & Desv. tip. \\
\hline C. Oral & 36,23 & 87 & 15,598 \\
\hline Odontopediatría & 32,05 & 78 & 17,129 \\
\hline Periodoncia & 41,41 & 73 & 14,306 \\
\hline Total & 36.45 & 238 & 16,117 \\
\hline
\end{tabular}


ron excelente en $33.3 \%$, regular en $29.9 \%$. ( $\mathrm{p}=, 190$; no hay significancia estadística).

En satisfacción con "comunicación con el dentista", la respuesta de pacientes de odontopediatría fue excelente en $48.7 \%$ y regular en $44.9 \%$. Los pacientes de periodoncia respondieron, excelente en $37.0 \%$ y regular en $41.1 \%$. Los pacientes de cirugía oral respondieron excelente en $44.8 \%$ y regular en $33.3 \%$. De acuerdo a la prueba de verosimilitud fue 0.018 ; es decir hay significancia estadística. Es mejor la comunicación con los pacientes de odontopediatría.

La satisfacción con "tiempo de espera en la clínica para acceder al dentista", la respuesta de pacientes de odontopediatría fue excelente en $23.1 \%$ y regular en $61.5 \%$. Los pacientes de periodoncia respondieron excelente en $42.5 \%$ y regular en $28.8 \%$. Los pacientes de cirugía oral respondieron excelente en $35.6 \%$, regular en $33.3 \%$. ( $p=, 001$; es decir hay una significancia estadística entre las especialidad, siendo mejor evaluada la especialidad de periodoncia).

Otros resultados se muestran en la Tabla 2.

\section{DISCUSIÓN}

Debemos estar concientes que esta encuesta no es suficiente para obtener resultados concluyentes de la satisfacción odontólogo-paciente, ya que debiéramos incorporar otros parámetros como ansiedad, temor, dolor, experiencias previas. No existe bibliografía nacional con trabajos en que se estudie satisfacción de pacientes con tres o más especialidades dentales en un mismo servicio.

Uno de los aspectos más valorados por los pacientes en estudios relacionados con este tema, está en el área de la comunicación e información por parte del dentista ${ }^{11}$. Los resultados encontrados en este estudio estuvieron en grados de satisfacción aceptable en esos factores, especialmente comunicación en odontopediatría, e información acerca de su tratamiento en cirugía oral. Algunos autores ${ }^{12,13}$ encuentran que los pacientes priorizan criterios que para ellos son extremadamente importantes, tales como explicación de los procedimientos, esterilización/higiene, habilidades del dentista, por sobre

Tabla 2. Respuesta "excelente" de las mujeres encuestadas, según especialidad expresada en porcentaje, en el servicio dental del Hospital de Rancagua, julio 2007.

\begin{tabular}{|l|c|c|c|c|}
\hline Pregunta & C.Oral & Odontopediatría & Periodoncia & Promedio \\
\hline Competencia técnica del dentista & 58.6 & 60.3 & 56.2 & 58.4 \\
\hline $\begin{array}{l}\text { Información acerca de su } \\
\text { tratamiento }\end{array}$ & 57.5 & 50.0 & 52.1 & 53.4 \\
\hline $\begin{array}{l}\text { Tiempo de espera en la clínica } \\
\text { para acceder al dentista }\end{array}$ & 35.6 & 23.1 & 42.5 & 33.6 \\
\hline Trato del dentista & 47.1 & 60.3 & 53.4 & 53.4 \\
\hline Comunicación con el dentista & 44.8 & 48.7 & 37.0 & 43.7 \\
\hline Costo del tratamiento & 29.9 & 50.0 & 60.3 & 45.8 \\
\hline Acceso al tratamiento dental & 32.2 & 46.1 & 58.9 & 44.9 \\
\hline Limpieza de la sala de espera & 54.0 & 53.8 & 49.3 & 52.5 \\
\hline Citaciones & 33.3 & 33.3 & 43.8 & 36.6 \\
\hline Disponibilidad de baños & 2.3 & 0 & 8.2 & 3.4 \\
\hline
\end{tabular}


equipos nuevos y decoración agradable. Para mejorar la satisfacción del paciente, es importante conocer lo que los pacientes quieren y no lo que los profesionales creen que debieran querer ${ }^{14,15}$.

La competencia técnica fue evaluada como excelente por el $60.3 \%$ de los pacientes de odontopediatría; $56.2 \%$ en periodoncia, y por el $58.6 \%$ de los pacientes de cirugía oral. Este factor fue el mejor evaluado por los pacientes de este estudio, que es a menudo citado como determinante de la satisfacción del paciente, pero es difícil de evaluar por el paciente ${ }^{16}$.

Algunos autores señalan que los pacientes, al evaluar la competencia técnica, la relacionan con la comunicación, trato e información entregada ${ }^{17}$. El tiempo de espera en la clínica para acceder al dentista y la obtención de citación en un día y hora determinado, tuvieron una evaluación bastante menos que regular por los pacientes y debe ser corregido. El tiempo de espera de los pacientes también está influenciado por el personal auxiliar, que es falente en el servicio dental del Hospital de Rancagua. Tenemos siete auxiliares paramédicos dentales para nueve clínicas de atención simultánea.

Algunos autores consideran como un factor importante en la satisfacción del paciente, la limpieza de la sala de espera, el confort de los asientos y música ambiental, que se ha demostrado influye en la evaluación de la satisfacción de los pacientes ${ }^{18}$. La limpieza de la sala de espera fue evaluada como excelente por el $52.5 \%$ de los pacientes en las tres especialidades de este estudio.

Algunos autores encuentran que las mujeres expresan mejor los niveles de satisfacción con la atención, que los hombres ${ }^{19}$.

Este trabajo tuvo el sesgo de que sólo considera la satisfacción de pacientes mujeres, debido a que las 238 pacientes encuestadas fueron mujeres. Es importante considerar que dos especialidades (cirugía oral y periodoncia) fueron de atención directa a las personas consultadas, mientras que en odontopediatría fue un tercero quien recibió la atención. Esta situación puede influenciar la percepción de algunos parámetros.

La disponibilidad de baños fue evaluada como muy mala, con un $72.7 \%$ por los pacientes de las tres especialidades en estudio, siendo peor evaluada por los pacientes de odontopediatría $(76.9 \%)$. Es totalmente justificada la evaluación de los pacientes debido a que no existen baños para los pacientes en las cercanías del servicio dental.

Al comparar la satisfacción del paciente con relación a las tres especialidades expuestas en este estudio, encontramos que la más deficiente es cirugía oral, en los aspectos evaluados relacionados con: trato del dentista, costo del tratamiento, acceso al tratamiento, obtención de citaciones en un tiempo determinado. Posiblemente se debe a la gran demanda por esta especialidad.

La satisfacción de los pacientes está basada en actitudes y sentimientos acerca del proveedor y de la atención obtenida. Por ello es importante la interrelación paciente-profesional ${ }^{20}$.

En opinión de los autores, en la calidad de la atención influyen factores indirectos, como los aspectos psicológicos que involucran las percepciones de los pacientes, quienes son los receptores de la atención dental. Este tipo de factor se traduce en sentimientos e incluyen malestar, frustración, dolor, miedo, desconfianza; que son opuestos en términos de satisfacción. En muchas oportunidades un manejo adecuado de estos factores por parte del dentista, evita considerablemente los reclamos de los pacientes y, por lo tanto, la evaluación de la calidad de la atención y su satisfacción, es mejor evaluada. Una explicación con un lenguaje adecuado, es siempre bien recibida por el paciente.

\section{CONCLUSIONES}

Los resultados indican que el nivel de satisfacción del paciente fue moderadamente excelente, en general. Áreas identificadas como necesitadas de mejoramiento incluyen: comunicación dentista-paciente, sistema de citación, acortar el tiempo de espera para acceder al dentista.

Destaca la gran insatisfacción manifestada por los pacientes por la escasa disponibilidad de baños. 
La medición de satisfacción del paciente es compleja de evaluar, debido a los múltiples factores que inciden y debe ser continua e incluir medidas de mejoramiento.

Recepción: 21 de diciembre de 2007 Aprobación: 8 de mayo de 2008

\section{REFERENCIAS}

1. MURRAY CJ, FRENK J. A framework for assessing the performance of health systems. Bull WHO. 2000; 78 (6):717-31.

2. WORLD HEALTH ORGANIZATION. Technical consultation on concepts and methods for measuring the responsiveness of health systems. En: Murray CJ, Evans DB, eds. Health systems performance assessment: debates, methods and empiricism. Geneva: World Health Organization; 2003. Pp. 115-23.

3. ZIMMERMAN R. The dental appointment and patient behavior. Differences in patients and practitioner preferences, patient satisfaction, and adherence. Med Care 1988; 26: 403-414.

4. BARNES N. Open wide: an examination of how patients select and evaluate their dentist. Health Market Q 1985; 3: 49-56.

5. GARFUNKEL E. The consumer speaks: How patients select and how much they know about dental health care personnel. J Prosthet Dent 1980; 43: 380-384.

6. MURRAY B, KAPLAN A. Patient satisfaction in 14 private dental practices. IDAR Abstract No. 892. J Dent Res 1981; 60: 532.

7. LAHTI S, HAUSEN H, KÄÄRIÄINEN R. Patients'expectations of an ideal dentist and their views concerning the dentist they visited: Do the views conform to the expectations and what determines how well they conform? Community Dent Oral Epidemiol 1996; 24: 240-244.
8. LIDDELL A, LOCKER D. Dental visit satisfaction in a group of adults aged 50 years and over. J Behav Med 1992; 15: 415-427.

9. LOCKER D, LIDDELLA. Correlates of dental anxiety among older adults. J Dent Res 1991; 70: 198-203.

10. LOCKER D, SHAPIRO D, LIDDELL A. Negative dental experiences and their relationship to dental anxiety. Community Dent Health 1996; 13: 86-92.

11. LAHTI S, TUUTI H, HAUSEN H, KÄÄRIÄINEN R. Comparison of ideal and actual behavior of patients and dentists during dental treatment. Community Dent Oral Epidemiol 1995; 23: 374-378.

12. GERBERT B, BLEECKER T, SAUB E. Dentists and the patients who love them: professional and patient views of dentistry. J Am Dent Assoc 1994; 125: 265272.

13. BURKE L, CROUCHER R. Criteria of good dental practice generated by general dental practitioners and patients. Int Dent J 1996; 46: 3-9.

14. ZIMMERMAN R. The dental appointment and patient behavior. Differences in patient and practitioner preferences, patient satisfaction, and adherence. Med Care 1988; 26: 403-414.

15. RAO C, ROSENBERG L. Consumer behavior analysis for improved dental services marketing. Health Market Q 1986; 3: 83-96.

16. ZEITHAML V, BITNER M. Services marketing. New York: McGraw-Hill, 1996.

17. CORAH N, O'SHEA R. Development of a patient measure of satisfaction with the dentist: The dental visit satisfaction scale. J Behav Med 1984; 7: 367373.

18. ANDRUS D, BUCHHEISTER J. Major factors affecting dental consumer satisfaction. Health Market Q 1985; 3: 57-68.

19. GOPALAKRISHNAP, MUMMALANENI V. Influencing satisfaction for dental services. J Health Care Market 1993; 13: 16-22.

20. HU T. The demand for dental care. In: Advances in Health Economic and Health Service Research, Vol II, Scheffer, RM. Ed. Jai Press 1981.

Usted puede comentar éste y otros artículos publicados en la Revista Chilena de Salud Pública, enviando un correo electrónico a revistasp@ med.uchile.cl 